\title{
Mercado Virtual móvel para estimulação cognitiva
}

\author{
Carlos Alexander de Pontes Medeiros, Luiz Paulo Baçal de Vasconcelos, \\ Rosa Maria E. Moreira da Costa
}

\author{
Departamento de Informática e Ciência da Computação \\ Universidade do Estado do Rio de Janeiro (UERJ) \\ Rio de Janeiro - Brazil \\ carlos_potencia@hotmail.com, luizv993@gmail.com, rcostalime.uerj.br
}

\begin{abstract}
Due to the high number of people with cognitive impairments and the increasing dispersion of mobile devices in society, we developed a virtual supermarket that proposes a series of challenges, with different levels of difficulties and objectives. At the end of each task, statistics are displayed, showing the user score. The main purpose of the system is to stimulate cognitive functions: working memory, reasoning and decision making. The system was evaluated by technological and health professionals and obtained positive results that stimulate the evaluation with end users in a next research stage.
\end{abstract}

Resumo. Em virtude do número elevado de pessoas com deficiências cognitivas e o aumento da dispersão de dispositivos móveis na sociedade, desenvolveu-se um ambiente de um supermercado virtual, que propõe uma série de desafios, com diferentes níveis de dificuldades e objetivos. Ao final de cada desafio, estatísticas são exibidas, mostrando a pontuação que o usuário conquistou naquela fase. O objetivo principal do sistema é estimular funções cognitivas, trabalhando a memória, raciocínio e tomada de decisões. O sistema foi avaliado por profissionais das áreas tecnológicas e da saúde e obteve resultados positivos, que estimulam a realização de avaliações com usuários finais como próxima etapa de pesquisa.

\section{Introdução}

O censo de 2010 do IBGE, apontou que $0,8 \%$ da população brasileira possui algum tipo de deficiência intelectual ou mental, sendo que $0,5 \%$ já nasceu com algum tipo de limitação [Vilela, 2015]. Os acidentes, sejam domésticos ou de trânsito, contribuem para aumentar essas estatísticas, sendo que em média 15\% das vítimas ficam com algum tipo de comprometimento das funções neurológicas [Jácomo, 2011]. Essas pessoas necessitam de uma rede de apoio que integra atividades de reabilitação física e neuropsiquiátrica, envolvendo diferentes tipos de atividades presenciais, ou a distância.

Em relação às sequelas cognitivas, várias iniciativas de exploração das novas tecnologias vêm sendo desenvolvidas, visando estimular funções cognitivas básicas, como atenção, concentração e memória, que são fundamentais para a realização das atividades de vida diária [Nunes,2018].

Recentemente, o crescimento das possibilidades das tecnologias móveis e das redes de comunicação ampliou o acesso e diminuiu os custos envolvidos no desenvolvimento e na utilização de jogos e aplicativos, principalmente, para as áreas da Saúde e Educação [Nunes,2018]. Segundo West et al. (2017), a aprendizagem móvel será explorada em um mundo mais conectado, onde a tecnologia será continuamente, mais acessível e barata. Essa expansão em nível mundial fomentou a criação de diferentes tipos de aplicações, voltadas para pessoas com necessidades especiais, verificando-se um considerável aumento no desenvolvimento de aplicações e jogos específicos para 
promover a estimulação de funções cognitivas de pessoas que foram atingidas por distúrbios e acidentes cerebrais [Matos, 2018], [Braz,2018].

Em geral, os supermercados oferecem várias possibilidades para desenvolver atividades que envolvem questões de aprendizado, estimulando diferentes funções cognitivas e executivas. Nesse ambiente, é possível treinar cálculo de valores, reconhecimento de marcas e produtos, reconhecimento de dinheiro e tomada de decisões, tão importantes para a realização de atividades de vida diária [van Herpen, 2016], [Josman, 2014]. Entretanto, existem poucas opções disponíveis para plataformas móveis e que tenham opções de classificação de níveis de dificuldade das atividades.

Logo, o objetivo deste trabalho é apresentar o desenvolvimento e os resultados de uma avaliação piloto de um ambiente de um supermercado, acessível em plataforma desktop e móvel, que estimula a atenção, a concentração e a tomada de decisões de pessoas com diferentes níveis de deficiências cognitivas, explorando graduações de dificuldade envolvidas no reconhecimento de produtos, moedas e valores.

Para atingir tais objetivos, o trabalho está dividido em seções. Esta Introdução, seguida da seção 2, que descreve alguns trabalhos correlatos, que foram fundamentais para o levantamento dos requisitos do sistema. A seção 3 descreve os requisitos e as tecnologias utilizadas. A seção 4 apresenta as telas, os resultados da avaliação piloto, seguida pelos Comentários Finais e as Referências.

\section{Trabalhos correlatos}

Visando identificar trabalhos relevantes relacionados ao uso de supermercados virtuais aplicados à área de reabilitação cognitiva apoiada no uso de tecnologias, realizouse uma pesquisa, destacando características a serem consideradas, ou não, na proposta de um novo sistema. A tabela1 apresenta algumas dessas características.

Tabela 1: Trabalhos que utilizam ambientes de supermercado aplicados em diferentes atividades relacionadas à estimulação cognitiva.

\begin{tabular}{|c|c|c|c|c|c|}
\hline Título & 2D & 3D & Plataforma & $\begin{array}{l}\text { Níveis de } \\
\text { dificuldade }\end{array}$ & Objetivo \\
\hline $\begin{array}{l}\text { Can a virtual } \\
\text { supermarket bring } \\
\text { realism into the lab? } \\
\text { Comparing shopping } \\
\text { behavior using virtual } \\
\text { and pictorial store } \\
\text { representations to } \\
\text { behavior in a physical } \\
\text { store [van Herpen, 2016] }\end{array}$ & $\mathrm{S}$ & $\mathrm{S}$ & Windows & $\mathrm{N}$ & $\begin{array}{l}\text { Comparar o comportamento } \\
\text { de usuários em um } \\
\text { supermercado virtual com o } \\
\text { comportamento em um } \\
\text { supermercado real }\end{array}$ \\
\hline $\begin{array}{l}\text { Using the Virtual Action } \\
\text { Planning-Supermarket } \\
\text { for Evaluating Executive } \\
\text { Functions in People with } \\
\text { Stroke [Josman, 2014] }\end{array}$ & $\mathrm{N}$ & $\mathrm{S}$ & Windows & $\mathrm{N}$ & $\begin{array}{l}\text { Comparar o desempenho dos } \\
\text { usuários utilizando } \\
\text { Planejamento de ações } \\
\text { Virtuais em um } \\
\text { supermercado }\end{array}$ \\
\hline $\begin{array}{l}\text { Using a virtual } \\
\text { supermarket as a tool for } \\
\text { training executive } \\
\text { functions in people with } \\
\text { mild cognitive } \\
\text { impairment } \\
\text { [Kizone,2012] }\end{array}$ & $\mathrm{N}$ & $S$ & Windows & $\mathrm{N}$ & $\begin{array}{l}\text { Verificar a eficácia do uso do } \\
\text { supermercado para melhorar } \\
\text { as funções cognitivas de } \\
\text { pessoas com problemas de } \\
\text { atenção e memória }\end{array}$ \\
\hline
\end{tabular}




\begin{tabular}{|c|c|c|c|c|c|}
\hline $\begin{array}{l}\text { Assessment of an } \\
\text { Immersive Virtual } \\
\text { Supermarket to Train } \\
\text { Post-stroke Patients: A } \\
\text { Pilot Study on Healthy } \\
\text { People } \\
\text { [Mondelline,2019] }\end{array}$ & $\mathrm{N}$ & $\mathrm{S}$ & $\begin{array}{l}\text { Usa } \\
\text { equipamento } \\
\text { imersivo } \\
\text { HTC VIVE }\end{array}$ & $\mathrm{N}$ & $\begin{array}{l}\text { Explorar o equipamento } \\
\text { imersivo para treinar pessoas } \\
\text { pós-Acidente Vascular } \\
\text { Cerebral }\end{array}$ \\
\hline
\end{tabular}

Como se observa na tabela 1 , todos os trabalhos têm em comum a estratégia de usar ambientes de supermercado como uma alternativa para apoiar o tratamento de pessoas com algum tipo de deficiência cognitiva. Observa-se, também, que nenhum dos trabalhos utilizou níveis de dificuldade em suas abordagens, e por isso esta funcionalidade foi considerada no projeto do "Mercado Virtual", pois um sistema que gera diferentes tipos de desafios pode estimular os usuários a se esforçarem para superá-los, ajudando na sua reabilitação. Observou-se que nenhum dos trabalhos desenvolveu a aplicação para plataformas móveis.

Todos os trabalhos estudados exploraram a tecnologia de realidade virtual, porém essa tecnologia dificulta a visualização e a navegação quando explorada em telas pequenas de smartphones, sem uso de equipamentos específicos para visualização 3D. E como o foco deste trabalho é proporcionar facilidade de uso, esses ambientes tridimensionais poderiam se mostrar muito complexos para pessoas com deficiências cognitivas.

Quanto aos níveis de dificuldade, mais especificamente, o uso de fases com níveis crescentes de complexidade pode gerar o desejo de superar os desafios, gerando satisfação quando a tarefa é finalizada, explorando abordagens de gamificação.

\section{Descrição e Tecnologias Adotadas}

Resumidamente, o "Mercado Virtual" tem como aspecto principal a acessibilidade do usuário e, para isso, foi desenvolvida uma aplicação híbrida que roda tanto em navegadores web (como o Google Chrome, por exemplo), para os usuários que prefiram utilizar o computador, quanto para smartphones Android, permitindo que o usuário possa utilizar o sistema a qualquer momento, sem necessidade sequer de conexão com a internet, pois o sistema pode ser executado totalmente off-line, já que é possível baixar todos os arquivos e dados necessários para o aparelho do usuário.

Os níveis de dificuldade do jogo vêm preencher uma lacuna percebida no estudo de trabalhos correlatos. Consideramos importante que haja uma sensação de progressão no jogo, onde o usuário tenha sempre um novo objetivo depois de finalizar um desafio, com níveis de dificuldade de crescimento bem lento, para que não cause frustrações nos usuários, em caso de insucesso na tarefa. Nesse caso, o número de itens requeridos em cada desafio vai aumentando e misturando itens de duas ou mais classes de produtos. Foi escolhida a visualização 2D para ampliar as possibilidades de acesso a um maior número de pessoas.

$\mathrm{Na}$ implementação do "Mercado Virtual" foram adotadas as seguintes tecnologias: HTML5, JavaScript, Jquery, PhoneGap, que é um framework usado para transformar aplicações web em aplicações móveis e vice-versa. Foi utilizado armazenamento local, um recurso do JavaScript, que armazena informações no browser do cliente, permitindo que toda vez que a aplicação for aberta, os dados sejam recuperados automaticamente. Foi usado ainda, a abordagem de "Aplicação Única": a aplicação não possui login e senha. Neste caso, os dados são salvos automaticamente, sendo associados 
à aplicação correspondente. Assim, o usuário não terá necessidade de memorizar nomes de usuário nem senhas.

\section{Descrição do Mercado Virtual}

O "Mercado Virtual" é dividido em setores, onde o usuário pode interagir usando o mouse (no caso de uso do desktop) ou toques na tela (no caso de smartphones) para realizar escolhas e superar os desafios que lhe são propostos.

A aplicação é baseada em fluxo de fases, com graus de dificuldade crescente, e o usuário vai tendo contato com atividade de vida diária, tais como: comprar uma lista de itens do supermercado; reconhecer produtos; identificar o valor das notas e moedas correntes e calcular valores. A cada fase é apresentada uma lista de compras que o usuário tem que comprar. Em seguida, ele tem que pagar o valor total da compra, identificando o valor das notas e moedas, que são apresentados na tela.

A seguir, são apresentadas algumas telas do "Mercado Virtual".
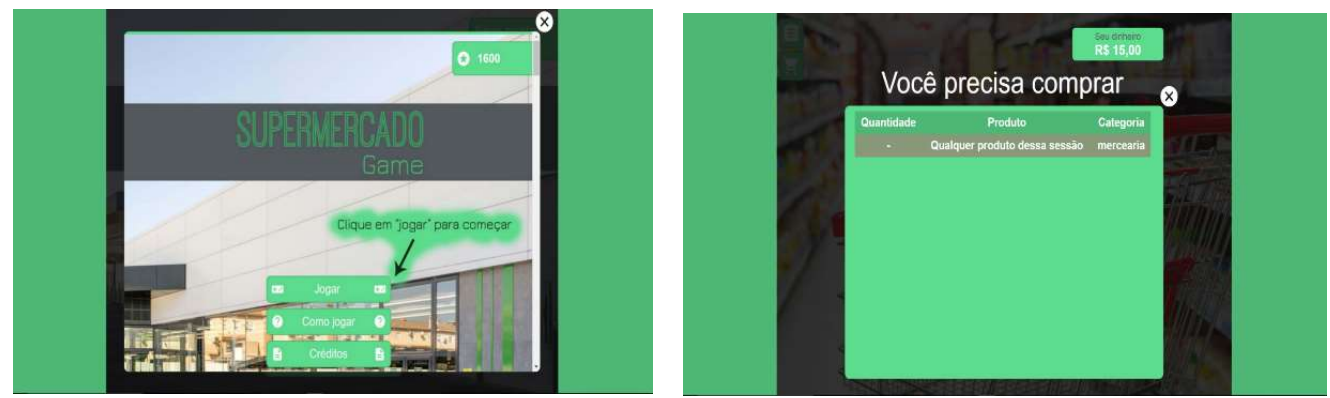

Figura 1a: Tela inicial, com a possibilidade de acessar tutorial; Figura 1b: tela de jogo, onde é apresentado o objetivo, contendo a lista com o tipo de produto a ser comprado, quantidade e categoria do produto; e no canto superior direito apresenta a quantia disponível para o usuário utilizar.

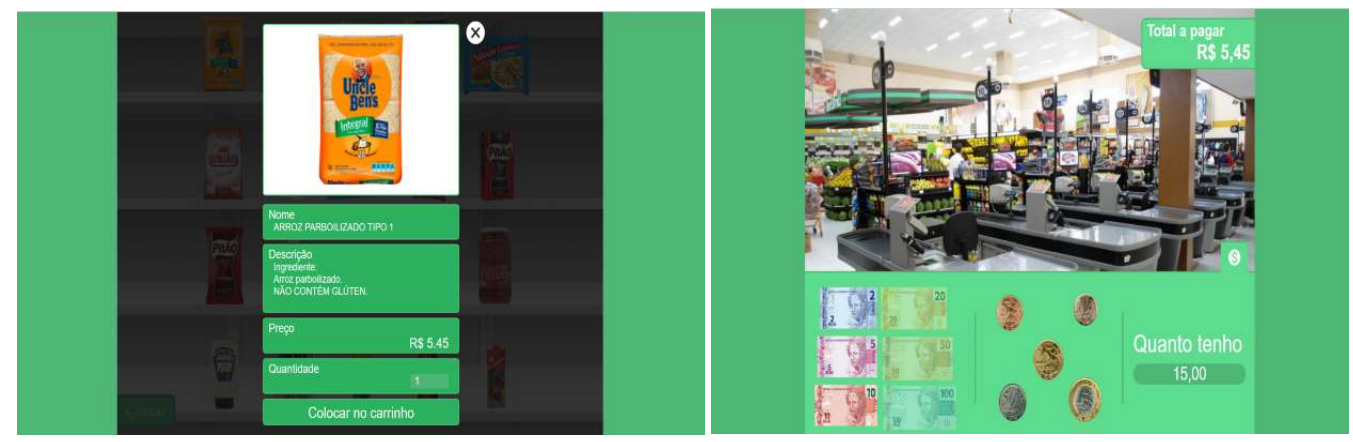

Figura 2a: a tela do produto contendo suas informações; Figura 2b: tela do caixa, que calcula o valor final da compra e onde são apresentadas as cédulas e moedas correntes. 0 usuário deve clicar sobre as notas e moedas e arrastar até o Caixa para pagar o produto.

O valor da pontuação será dado de acordo com os objetivos concluídos: quanto mais objetivos concluídos e mais próximo do total a pagar for a quantia escolhida pelo jogador, maior será a pontuação. 


\subsection{Avaliação Piloto}

Uma avaliação piloto foi realizada com 8 participantes, com média de idade de 32 anos, variando de 22 a 55 anos, sendo $50 \%$ do sexo masculino e $50 \%$ do sexo feminino, sendo 3 participantes da área médica e 5 da área tecnológica.

Cada um deles usou a aplicação do "Mercado Virtual" e em seguida, respondeu a um questionário, disponibilizado via web, composto de duas partes: uma associada a critérios de usabilidade e outra específica para profissionais da área da saúde. $\mathrm{O}$ questionário foi composto por afirmativas, que eram classificadas segundo uma escala de Likert com 5 níveis. Em relação à usabilidade, os resultados são apresentados na tabela 2 .

Tabela 2: Resultados do estudo piloto relacionados a questões de usabilidade

\begin{tabular}{|l|c|c|c|c|c|}
\hline & $\begin{array}{l}\text { Concordo } \\
\text { Fortemente }\end{array}$ & $\begin{array}{l}\text { Concordo } \\
\text { Fracamente }\end{array}$ & Neutro & $\begin{array}{l}\text { Discordo } \\
\text { Fracamente }\end{array}$ & $\begin{array}{l}\text { Discordo } \\
\text { Fortemente }\end{array}$ \\
\hline A aplicação é fácil de usar & $62,5 \%$ & $25 \%$ & $12,5 \%$ & & \\
\hline A navegação é facil & $37,5 \%$ & $50 \%$ & & $12,5 \%$ & \\
\hline As cores são agradáveis & $37,5 \%$ & $50 \%$ & $12,5 \%$ & & \\
\hline O tempo de resposta é adequado & $75 \%$ & $12,5 \%$ & $12,5 \%$ & & \\
\hline $\begin{array}{l}\text { Os objetos que compõe as cenas } \\
\text { são adquados }\end{array}$ & $50 \%$ & $37,5 \%$ & $12,5 \%$ & & \\
\hline As cenas são realistas & $50 \%$ & $12,5 \%$ & $25,5 \%$ & & $12,5 \%$ \\
\hline
\end{tabular}

Uma segunda parte do formulário era específica para profissionais da área da saúde, visando avaliar a utilidade, cujos resultados são apresentados na tabela 3 .

Tabela 3: Resultados do estudo piloto relacionados à utilidade do produto

\begin{tabular}{|c|c|c|c|c|c|}
\hline & $\begin{array}{l}\text { Concordo } \\
\text { Fortemente }\end{array}$ & $\begin{array}{l}\text { Concordo } \\
\text { Fracamente }\end{array}$ & Neutro & $\begin{array}{l}\text { Discordo } \\
\text { Fracamente }\end{array}$ & $\begin{array}{l}\text { Discordo } \\
\text { Fortemente }\end{array}$ \\
\hline $\begin{array}{l}\text { As atividades propostas são } \\
\text { relevantes para pacientes } \\
\text { neuropsiquiátricos }\end{array}$ & $66,7 \%$ & & & $33,3 \%$ & \\
\hline $\begin{array}{l}\text { O software é adequado para ser } \\
\text { utilizado no processo de } \\
\text { estimulação cognitiva }\end{array}$ & $100 \%$ & & & & \\
\hline $\begin{array}{l}\text { Os níveis de dificuldade são } \\
\text { adequados para serem utilizados } \\
\text { com pacientes neuropsiquiátricos }\end{array}$ & $33,3 \%$ & $33,3 \%$ & $33,3 \%$ & & \\
\hline $\begin{array}{l}\text { Eu usaria o software com meus } \\
\text { pacientes }\end{array}$ & $33,3 \%$ & $33,3 \%$ & & $33,3 \%$ & \\
\hline
\end{tabular}

Os resultados destacam um nível alto de satisfação em relação à usabilidade do produto. Mas, observa-se um ponto a ser ressaltado: todos os participantes da área da saúde consideraram que a aplicação é adequada para ser utilizada em processos de Reabilitação Cognitiva.

\section{Comentários Finais}

Este trabalho teve por objetivo desenvolver e avaliar um aplicativo que explora atividades realizadas em supermercados, para estimular processos de atenção, concentração e memória de pessoas com diferentes tipos de deficiências cognitivas.

A experiência de desenvolver algo voltado para um público com déficit cognitivo se mostrou um desafio interessante, que envolveu estudos teóricos sobre os processos cognitivos e estudos que identificaram trabalhos correlatos, contendo características importantes em relação a atividades e tecnologias capazes de manter a atenção e o interesse dos pacientes. O desejo de que o aplicativo pudesse funcionar em plataformas 
desktop e em smartphones limitou a proposição de algumas atividades, de forma que elas pudessem não perder qualidade gráfica, quando utilizadas em smartphones.

A estratégia de associar desafios e pontuações veio ao encontro dos estudos sobre o uso de técnicas de gamificação, que ressaltam um aumento da motivação e do engajamento dos usuários nas atividades, quando existe algum tipo de competição implícita [Klock, 2014]. Com isso podemos atingir os objetivos da atividade, sem que o usuário perceba sua relação com atividades terapêuticas.

A avaliação piloto foi limitada a um número restrito de participantes, mas apontou um bom nível de satisfação com interesse de profissionais da área da saúde em utilizar o software com seus pacientes.

Como trabalhos futuros, espera-se atualizar alguns aspectos associados à interface do software, para então, submeter o projeto ao Comitê de Ética e realizar uma avaliação com um número significativo de profissionais da área da saúde.

O software está disponível em: http://playerdois.com.br/luiz/projeto/. O tutorial é acessado no botão "Como jogar", utilizando a barra de rolagem.

\section{Referências}

Braz, Priscilla ; Werneck, Vera M. B. ; de Souza Cunha, Herbet ; Costa, Rosa M. E. Moreira. (2018) SMEC-3D: A Multi-agent 3D Game to Cognitive Stimulation. In: Bajo J. et al.. (Org.). Communications in Computer and Information Science. 1ed.: Springer International Publishing, v. 887 , p. $247-258$.

Jácomo, A.A. E.; Garcia, A.C. F. (2011) Análise dos acidentes motociclísticos no Centro de Reabilitação e Readaptação Dr. Henrique Santillo (CRER). ActaFisiatrica, v. 18, n. 3.

Josman, N., Kizony, R., Hof, E., Goldenberg, K., Weiss, P. L., \& Klinger, E. (2014). Using the virtual action planning-supermarket for evaluating executive functions in people with stroke. Journal of Stroke and Cerebrovascular Diseases, v. 23, n.5, p. 879-887.

Kizony, R., Korman, M., Sinoff, G., Klinger, E., Josman, N., Sharky, P. M., \& Klinger, E. (2012). Using a virtual supermarket as a tool for training executive functions in people with mild cognitive impairment. In Proceedings of the 9th International Conference on Disability, Virtual Reality and Associated Technologies, Reading, England, p. 41-46.

Klock, Ana Carolina et al., (2014) Análise das Técnicas de Gamificação em Ambientes Virtuais de Aprendizagem, RENOTE, v.12, n. 2.

Matos, L. F., Santos, M. H., Boechat, Y., Amorim, G., \& Muchaluat-Saade, D. C. (2018). MemoGinga: Jogo Interativo para TV Digital como Exercício Cognitivo para Idosos. In Anais do XVIII Simpósio Brasileiro de Computação Aplicada à Saúde. SBC.

Mondellini, M., Pizzagalli, S., Greci, L., Sacco, M. (2019). Assessment of an Immersive Virtual Supermarket to Train Post-stroke Patients: A Pilot Study on Healthy People. International Conference on Augmented Reality, Virtual Reality and Computer Graphics.Springer,p.313-329.

Nunes, F. L. S., Oliveira, J. C., Machado, L. S., Nunes, E. P. S., Costa, Rosa M. E. M.; Queiroz, A. C. M. . Capítulo 15 - Saúde. In: Romero Tori; Marcelo da Silva Hounsell. (Org.). Introdução a Realidade Virtual e Aumentada. 1ed.Porto Alegre: Editora SBC, 2018, v. 1, p. 839-940.

van Herpen, E., van den Broek, E., van Trijp, H. C., \& Yu, T. (2016). Can a virtual supermarket bring realism into the lab? Comparing shopping behavior using virtual and pictorial store representations to behavior in a physical store. Appetite, v. 107, p. 196-207.

Vilela, F. (2015) IBGE: 6,2\% da população têm algum tipo de deficiência. Disponível em: https://agenciabrasil.ebc.com.br/geral/noticia/2015-08/ibge-62-da-populacao-tem-algum-tipode-deficiencia, visitado em abril 2020.

West, M.; Winters, N.; Shuler, C. (2014) O Futuro da Aprendizagem Móvel - Implicações para Planejadores e Gestores de Política. UNESCO. Disponível em: http://unesdoc.unesco.org/images/0022/002280/228074POR.pdf, visitado em abril 2020. 